\title{
Thiazide-Induced Hyperglycemia in a 6 Years Old Girl: A Case Report and Review of the Literature
}

\author{
Hina Walia ${ }^{\mathrm{a}, \mathrm{c}}$, Joseph D. Tobias ${ }^{\mathrm{a}, \mathrm{b}}$
}

\begin{abstract}
Various factors may affect glucose homeostasis in children resulting in rare clinical scenarios. Alterations in glucose regulation and hyperglycemia may result from pharmacologic interventions and the introduction of new medications. Although used most commonly to control fluid balance, an uncommon adverse effect of the thiazide diuretics is hyperglycemia. We present a 6-year-old girl with congenital heart disease who developed clinically significant hyperglycemia when a thiazide diuretic was added to her medication regimen. Previous reports of similar responses are reviewed, physiologic mechanisms are postulated, and treatment strategies are proposed.
\end{abstract}

Keywords: Hyperglycemia; Thiazides; Diazoxide

\section{Introduction}

Various factors may be responsible for alterations in glucose homeostasis in children. In rare clinical scenarios, alterations in glucose regulation and hyperglycemia may result from pharmacologic interventions and the introduction of new medications [1-5]. Although used most commonly to control fluid balance, an uncommon adverse effect of the thiazide diuretics is hyperglycemia. We present a 6-year-old girl with congenital heart disease (CHD) who developed profound hyperglycemia when a thiazide diuretic was added to her medication regimen. Previous reports of similar responses are reviewed, physiologic mechanisms are postulated, and treatment strategies are proposed.

Manuscript accepted for publication January 07, 2016

aDepartment of Anesthesiology \& Pain Medicine, Nationwide Children's Hospital, Columbus, $\mathrm{OH}$, USA

bepartment of Anesthesiology \& Pain Medicine, The Ohio State University, Columbus, OH, USA

${ }^{\mathrm{c} C o r r e s p o n d i n g ~ A u t h o r: ~ H i n a ~ W a l i a, ~ D e p a r t m e n t ~ o f ~ A n e s t h e s i o l o g y ~ \& ~ P a i n ~}$ Medicine, Nationwide Children's Hospital, 700 Children's Drive, Columbus, OH 43205, USA. Email: hina.walia@nationwidechildrens.org

doi: http://dx.doi.org/10.14740/jmc2403w

\section{Case Report}

Institutional Review Board approval is not required at Nationwide Children's Hospital (Columbus, $\mathrm{OH}$ ) for the presentation of single case reports. The patient was a 6.5 -year-old, $17.75 \mathrm{~kg}$ girl who was sent to the emergency department after clinical blood work revealed an elevated blood glucose level. She had originally been brought in for outpatient blood work 1 week after the addition of an additional diuretic, chlorothiazide, to her medication regimen. The laboratory analysis at the outpatient clinic revealed a blood glucose level greater than $700 \mathrm{mg} /$ $\mathrm{dL}$. Additional complaints included that she seemed more tired than usual and there had been a $2.1 \mathrm{~kg}$ weight loss from the prior week's appointment. There was no history of other constitutional symptoms such as fever, cough or diarrhea. Her past medical history was significant for a congenital hyperinsulin state controlled with oral diazoxide, hypoplastic left heart syndrome (HLHS), and coarctation of the aorta. Her past surgical history included multiple cardiac procedures for surgical palliation of HLHS including: a stage 1 hybrid procedure in 2009 which was followed by balloon atrial septostomy 6 - 8 weeks later, surgical atrial septectomy later that year, and a modified comprehensive stage II procedure with a Glenn shunt, followed most recently by a Fontan procedure in 2015 [6]. The Fontan procedure was complicated by a chronic pleural effusion requiring an implanted pleural catheter. Other associated co-morbid conditions included gastroesophageal reflux, a seizure disorder, previous hypoxic-ischemic insults, developmental delay, and hypotonia. Her home medications included spironolactone $20 \mathrm{mg}$ twice a day, aspirin $81 \mathrm{mg}$ once a day, diazoxide 50 $\mathrm{mg}$ three times a day as well as the recently added chlorothiazide $250 \mathrm{mg}$ once a day. There were no known drug allergies. Following admission to the Cardiothoracic ICU (CTICU), the blood glucose values remained elevated ranging from 569 to $702 \mathrm{mg} / \mathrm{dL}$ along with low sodium and chloride values. The venous blood gas at the time of admission to the CTICU showed a normal $\mathrm{pH}$ and normal lactate levels $\left(\mathrm{pH} 7.36, \mathrm{PaCO}_{2} 52\right.$ $\mathrm{mm} \mathrm{Hg}, \mathrm{PaO}_{2} 47 \mathrm{mmHg}, \mathrm{HCO}_{3} 22 \mathrm{mmol} / \mathrm{L}$, base excess +3.3 $\mathrm{mmol} / \mathrm{L}$, and lactate $1.3 \mathrm{mmol} / \mathrm{L}$ ). The chlorothiazide and diazoxide were stopped; however, the blood glucose values did not return to normal so an intravenous insulin infusion was started at 0.02 units $/ \mathrm{kg} / \mathrm{h}$. This was continued for 3 days which resulted in control of her blood glucose values. Her insulin regimen was then transitioned to intermittent subcutaneous insulin 
for 1 week at which time chlorothiazide was restarted. Following the restarting of chlorothiazide, the insulin therapy was slowly withdrawn without any clinically significant change in her blood glucose values. The remainder of the hospital stay was uneventful with close monitoring of blood glucose values which remained within normal limits. The patient was discharged home 18 days after the initial admission date.

\section{Discussion}

Hyperglycemia in children may present simply as increased thirst, frequent urination, and fatigue or in the later stages as severe as nausea, vomiting, alterations in the pattern of respiration (Kussmaul breathing), abdominal pain, and even coma. While there are several potential causes of hyperglycemia, the most common in children is a decrease in the production of insulin by the pancreas which results in diabetes mellitus (DM). DM is generally classified as type 1 or type 2 . Type $1 \mathrm{DM}$ is the result of an autoimmune process with destruction of the insulin-producing beta cells of the pancreas. In contrast, various pathophysiologic processes contribute to type 2 DM including varying degrees of insulin resistance and relative insulin deficiency [7-11]. The clinical features of DM may be modified by genetic or environmental influences.

There are major metabolic derangements that may occur in patients with either DM type 1 or type 2. Diabetic ketoacidosis (DKA) generally occurs as a complication of DM type 1 and hyperosmolar hyperglycemia (HHS) as a complication of DM type 2 [12]. Both of these are life-threatening emergencies requiring hospitalization and aggressive therapy to reduce morbidity and mortality. A hyperosmolar hyperglycemic state, as was noted in our patient, was previously known as hyperosmolar hyperglycemic non-ketotic coma (HHNC). It is a less common consequence of profound hyperglycemia, seen most commonly in the adult patient with DM type 2. The terminology was changed because coma occurred in less than $20 \%$ of patients. HHS most commonly occurs in patients with type 2 DM who have a concomitant illness that leads to reduced fluid intake. HHS is characterized by hyperglycemia, hyperosmolarity and dehydration without ketoacidosis; however, it carries a higher mortality rate than DKA estimated at approximately $10-20 \%$. Most patients present with severe dehydration and focal or global neurological deficits. In as many as one-third of patients, the clinical features of HHS and DKA overlap. In our patient, hyperglycemia and subsequently HHS coincided with initiation of therapy with the diuretic, chlorothiazide. This interaction has been documented by other reports $[4,13-$ 16]. Treatment with thiazide diuretics has been shown to be an independent predictor of the onset of new diabetes among patients with hypertension [4]. While the exact process responsible for the hyperglycemia associated with the thiazides diuretics remains speculative, it has been postulated that they may increase serum glucose values by activating the renin/angiotensin/aldosterone and sympathetic nervous systems [17, 18]. These mechanisms are supported by the fact that the effects of thiazides on serum glucose can be mitigated by inhibiting the renin/angiotensin/aldosterone axis. Alternatively, it has been suggested that hypokalemia causes an indirect reduction in insulin secretion, thereby leading others to suggest strategies that maintaining normal potassium concentrations may prevent glucose intolerance $[4,17,18]$.

In summary, we present the development of clinically significant hyperglycemia following the addition of a thiazide diuretic to the medication regimen of a 6-year-old girl with associated CHD. The clinical consequences were likely magnified by the accompanying administration of diazoxide to treat a previously diagnosed hyperinsulin state. Once the diazoxide was discontinued, it was then feasible to reinstitute chlorothiazide therapy without alterations in serum glucose levels. Given this and additional adverse effects of the thiazide class of medications, other agents are generally used as first-line treatment of hypertension in adults. Although uncommon, when such effects are seen in the pediatric population, alternative diuretics should be used. Alternatively, it appears that the response may be mitigated by maintaining the serum potassium value within the normal range through the use of potassium-sparing diuretics and/or oral potassium supplementation. In specific patient populations, close monitoring of blood glucose values may be required.

\section{References}

1. Bleicher SJ, Chowdhury F, Podolsky S, Fleishman P, Goldner MG. Studies on diazoxide-induced hyperglycemia: an extrapancreatic mechanism. Ann N Y Acad Sci. 1968;150(2):294-302.

2. Carter BL. Preventing thiazide-induced hyperglycemia: opportunities for clinical pharmacists. Pharmacotherapy. 2008;28(12):1425-1428.

3. Carter BL, Basile J. Development of diabetes with thiazide diuretics: the potassium issue. J Clin Hypertens (Greenwich). 2005;7(11):638-640.

4. Zillich AJ, Garg J, Basu S, Bakris GL, Carter BL. Thiazide diuretics, potassium, and the development of diabetes: a quantitative review. Hypertension. 2006;48(2):219224.

5. Fathallah N, Slim R, Larif S, Hmouda H, Ben Salem C. Drug-Induced Hyperglycaemia and Diabetes. Drug Saf. 2015;38(12):1153-1168.

6. Naguib AN, Winch P, Schwartz L, Isaacs J, Rodeman R, Cheatham JP, Galantowicz M. Anesthetic management of the hybrid stage 1 procedure for hypoplastic left heart syndrome (HLHS). Paediatr Anaesth. 2010;20(1):38-46.

7. Roizen JD, Bradfield JP, Hakonarson H. Progress in understanding type 1 diabetes through its genetic overlap with other autoimmune diseases. Curr Diab Rep. 2015;15(11):102.

8. Stumvoll M, Goldstein BJ, van Haeften TW. Type 2 diabetes: principles of pathogenesis and therapy. Lancet. 2005;365(9467):1333-1346.

9. Beck-Nielsen H, Groop LC. Metabolic and genetic characterization of prediabetic states. Sequence of events leading to non-insulin-dependent diabetes mellitus. J Clin Invest. 1994;94(5):1714-1721.

10. Kahn CR. Banting Lecture. Insulin action, diabe- 
togenes, and the cause of type II diabetes. Diabetes. 1994;43(8):1066-1084.

11. Robertson RP. Antagonist: diabetes and insulin resistance--philosophy, science, and the multiplier hypothesis. J Lab Clin Med. 1995;125(5):560-564; discussion 565.

12. Pasquel FJ, Umpierrez GE. Hyperosmolar hyperglycemic state: a historic review of the clinical presentation, diagnosis, and treatment. Diabetes Care. 2014;37(11):31243131.

13. Amery A, Berthaux P, Bulpitt C, Deruyttere M, de Schaepdryver A, Dollery C, Fagard R, et al. Glucose intolerance during diuretic therapy. Results of trial by the European Working Party on Hypertension in the Elderly. Lancet. 1978;1(8066):681-683.

14. Savage PJ, Pressel SL, Curb JD, Schron EB, Applegate WB, Black HR, Cohen J, et al. Influence of long-term, low-dose, diuretic-based, antihypertensive therapy on glucose, lipid, uric acid, and potassium levels in older men and women with isolated systolic hypertension:
The Systolic Hypertension in the Elderly Program. SHEP Cooperative Research Group. Arch Intern Med. 1998;158(7):741-751.

15. Tweeddale MG, Ogilvie RI, Ruedy J. Antihypertensive and biochemical effects of chlorthalidone. Clin Pharmacol Ther. 1977;22(5 Pt 1):519-527.

16. Lakshman MR, Reda DJ, Materson BJ, Cushman WC, Freis ED. Diuretics and beta-blockers do not have adverse effects at 1 year on plasma lipid and lipoprotein profiles in men with hypertension. Department of Veterans Affairs Cooperative Study Group on Antihypertensive Agents. Arch Intern Med. 1999;159(6):551-558.

17. Ellison DH, Loffing J. Thiazide effects and adverse effects: insights from molecular genetics. Hypertension. 2009;54(2):196-202.

18. Kudoh T, Nagawaga T, Nakagawa I. Additional small amounts of diuretics improve blood pressure control at low cost without disadvantages in blood sugar metabolism. Hypertens Res. 2008;31(3):455-462. 